Ingelheim, Bristol Myers Squibb, Celgene, Eli Lilly, Galapagos, GlaxoSmithKline, Janssen, Novartis, Pfizer, UCB, Grant/research support from: AbbVie, Eli Lilly, GlaxoSmithKline, Novartis, Pfizer, UCB, Désirée van der Heijde Consultant of: AbbVie, Amgen, Astellas, AstraZeneca, Bayer, Bristol Myers Squibb, Boehringer Ingelheim, Celgene, Cyxone, Daiichi, Eisai, Eli Lilly, Galapagos, Gilead, GlaxoSmithKline, Janssen, Merck, Novartis, Pfizer, Regeneron, Roche, Sanofi, Takeda, UCB Pharma. Director of Imaging Rheumatology BV, Frank Behrens Consultant of: Pfizer, AbbVie, Sanofi, Lilly, Novartis, Genzyme, Boehringer, Janssen, MSD, Celgene, Roche, Chugai, Bristol Myers Squibb, UCB Pharma, Grant/research support from: Pfizer, Janssen, Chugai, Celgene, Roche, Alan Kivitz Shareholder of: Pfizer, Sanofi, GlaxoSmithKline, Gilead Sciences, Inc., Novartis; Paid consultant: AbbVie, Boehringer Ingelheim, Flexion, Janssen, Pfizer, Sanofi, Regeneron, SUN Pharma Advanced Research, Gilead Sciences, Inc, Speakers bureau: Amgen, Horizon, Lilly, Novartis, Pfizer, Sanofi, Genzyme, Flexion, AbbVie, Thomas Lehman Shareholder of: Bristol Myers Squibb, Employee of: Bristol Myers Squibb, Lan Wei Shareholder of: Bristol Myers Squibb, Employee of: Bristol Myers Squibb, Marleen Nys Shareholder of: Bristol Myers Squibb, Employee of: Bristol Myers Squibb, Subhashis Banerjee Shareholder of: Bristol Myers Squibb, Employee of: Bristol Myers Squibb, Miroslawa Nowak Shareholder of: Bristol Myers Squibb, Employee of: Bristol Myers Squibb

DOI: 10.1136/annrheumdis-2021-eular.2653

\section{OP0228 \\ EFFICACY AND SAFETY OF RISANKIZUMAB FOR ACTIVE PSORIATIC ARTHRITIS, INCLUDING PATIENTS WITH INADEQUATE RESPONSE OR INTOLERANCE TO BIOLOGIC THERAPIES: 24-WEEK RESULTS FROM THE PHASE 3, RANDOMIZED, DOUBLE-BLIND, KEEPSAKE 2 TRIAL}

A. Ostor ${ }^{1}$, F. Van den Bosch ${ }^{2}$, K. Papp ${ }^{3}$, C. Asnal ${ }^{4}$, R. Blanco ${ }^{5}$, J. Aelion ${ }^{6}$, G. Alperovich ${ }^{7}$, Y. Zhang ${ }^{8}$, Z. Wang ${ }^{8}$, A. M. Soliman ${ }^{8}$, A. Eldred ${ }^{8}$, A. Kivitz ${ }^{9}$. ${ }^{1}$ Monash University, Cabrini Hospital, and Emertius Research, Melbourne, Australia; ${ }^{2}$ Ghent University, Department of Rheumatology, Gent, Belgium ${ }^{3}$ Probity Medical Research, K. Papp Clinical Research, Waterloo, Canada; ${ }^{4}$ Buenos Aires, DOM Centro de Reumatología, Buenos Aires, Argentina; ${ }^{5}$ Hospital Universitario Marqués de Valdecilla, IDIVAL, Santander, Spain; ${ }^{6}$ West Tennessee Research Institute, Not Applicable, Jackson, United States of America; ${ }^{7}$ AbbVie, Inc, Not Applicable, Madrid, Spain; ${ }^{8}$ AbbVie, Inc, Not Applicable, North Chicago, United States of America; ${ }^{9}$ Altoona Center for Clinical Research, Not Applicable, Duncansville, United States of America

Background: Risankizumab (RZB) is a humanized immunoglobin G1 monoclonal antibody that specifically inhibits interleukin 23 by binding its $p 19$ subunit. RZB is being investigated as a treatment for adults with psoriatic arthritis (PsA). Objectives: To compare the efficacy and safety of RZB vs placebo (PBO) for the treatment of active PsA in patients who have had inadequate response or intolerance to 1 or 2 biologic therapies (Bio-IR) or to $\geq 1$ conventional synthetic disease modifying antirheumatic drug (cSDMARD-IR).

Methods: KEEPsAKE 2 (NCT03671148) enrolled adults with active PsA ( $\geq$ 5 swollen joints [SJC] and $\geq 5$ tender joints [TJC]) who were Bio-IR or cSDMARD-IR. Patients were randomized to receive blinded subcutaneous RZB $150 \mathrm{mg}$ or $\mathrm{PBO}$ at weeks 0,4 , and 16 . The primary endpoint was the proportion of patients achieving $\geq 20 \%$ improvement in American College of Rheumatology score (ACR20) at week 24. Ranked secondary endpoints and other secondary endpoints are shown in the Table. Safety was assessed throughout the study. Results reported here are from the 24-week double-blind period; the open-label period with all patients receiving RZB is ongoing. Results: A total of 443 patients (RZB, $N=224 ; \mathrm{PBO}, \mathrm{N}=219$ ) were included in the analysis. Demographics and baseline disease characteristics were similar across treatment arms (mean SJC: 13.3; mean TJC: 22.6; mean duration of PsA: 8.2 years; mean body surface area involved with psoriasis $[B S A]$ in patients with BSA $\geq 3 \%: 12.1 \%)$; 206 patients $(46.5 \%)$ were Bio-IR. Significantly greater proportions of RZB-treated patients vs PBO-treated patients achieved the primary endpoint ( $51.3 \%$ vs $26.5 \%$, respectively; $P<.001)$ and all ranked secondary endpoints $(P<.001$ for all except for Functional Assessment of Chronic Illness Therapy-Fatigue [FACIT-Fatigue; $P<.009$ ]; Table). Other secondary outcomes also showed improvement for RZB- vs PBO-treated patients (Table). Serious adverse events were reported for $4.0 \%$ and $5.5 \%$ of RZB- and PBO-treated patients, respectively; serious infections were reported for $0.9 \%$ and $2.3 \%$.

Conclusion: RZB resulted in significantly greater improvements in signs and symptoms of PsA compared with PBO and was well tolerated in patients who were Bio-IR or csDMARD-IR.
Table. Efficacy Results

\begin{tabular}{|c|c|c|c|c|}
\hline & $\begin{array}{c}\text { RZB } 150 \mathrm{mg} \\
\mathrm{N}=224\end{array}$ & $\begin{array}{c}\text { PBO } \\
N=219\end{array}$ & $\begin{array}{c}\text { Difference } \\
(95 \% \mathrm{Cl})\end{array}$ & $P$ value ${ }^{\mathrm{a}}$ \\
\hline \multicolumn{5}{|l|}{ Primary endpoint } \\
\hline ACR20, \% & 51.3 & 26.5 & $24.5(15.9,33.0)$ & $<.001^{* * *}$ \\
\hline \multicolumn{5}{|l|}{ Ranked secondary endpoints } \\
\hline HAQ-DI score, change & -0.22 & -0.05 & $-0.16(-0.26,-0.07)$ & $<.001^{\star \ldots *}$ \\
\hline PASI 90,a $\%$ & 55.0 & 10.2 & $44.3(33.9,54.6)$ & $<.001^{* \ldots *}$ \\
\hline ACR20 at week $16, \%$ & 48.3 & 25.3 & $22.6(13.9,31.2)$ & $<.001^{* \times *}$ \\
\hline MDA, \% & 25.6 & 11.4 & $14.0(7.0,21.0)$ & $<.001^{\star \star * *}$ \\
\hline SF-36 PCS score, change & 5.9 & 2.0 & $3.9(2.4,5.3)$ & $<.001^{\star \star *}$ \\
\hline FACIT-Fatigue score, change & 4.9 & 2.6 & $2.2(0.6,3.9)$ & $.009^{\star \star}$ \\
\hline \multicolumn{5}{|l|}{ Other secondary endpoints } \\
\hline ACR50, \% & 26.3 & 9.3 & $16.6(9.7,23.6)$ & $<.001^{\dagger}$ \\
\hline ACR70, \% & 12.0 & 5.9 & $6.0(0.8,11.3)$ & $.024^{\dagger}$ \\
\hline Resolution of enthesitis, ${ }^{a} \%$ & 42.9 & 30.4 & $13.8(3.5,24.2)$ & $.009^{\dagger}$ \\
\hline Resolution of dactylitis, ${ }^{a} \%$ & 72.5 & 42.1 & $38.8(22.9,54.8)$ & $<.001^{\dagger}$ \\
\hline $\begin{array}{l}\text { ACR20/ACR50/ACR70, } \geq 20 / 50 / 70 \% \text { im } \\
\text { Functional Assessment of Chronic llines } \\
\text { Index; MDA, minimal disease activity, P, } \\
\text { RZB, risankizumab; SF-36 PCS, 36-Iten } \\
\text { All endpoints assessed at week } 24 \text { unles } \\
\cdots \text { : statistically significant at } 0.001 \text { level, } \\
\cdots: \text { Statistically significant at } 0.01 \text { level; } \\
\text { i: Nominal P value. }\end{array}$ & $\begin{array}{l}\text { ovement in America } \\
\text { Therapy-Fatigue; HA } \\
90, \geq 90 \% \text { reductic } \\
\text { Short Form Health S } \\
\text { otherwise noted. All } \\
\text { hultiplicity controlled. } \\
\text { Itiplicity controlled. }\end{array}$ & $\begin{array}{l}\text { in College of } \\
\text { AQ-DI, Healt } \\
\text { on in Psorias } \\
\text { survey Physic } \\
\text { "l changes ar }\end{array}$ & $\begin{array}{l}\text { Aseumatology score; FACIT-F } \\
\text { Assessment Questionnaire- } \mathrm{C} \text { Area Severity Index; PBO, } \\
\text { I Component Summary. } \\
\text { mean changes from baseline }\end{array}$ & $\begin{array}{l}\text { Eatigue, } \\
\text { Disability } \\
\text { lacebo; }\end{array}$ \\
\hline
\end{tabular}

$P B O N=158)$, or dactylitis $(R Z B N=40 ; P B O N=57)$ at baseline.

Disclosure of Interests: Andrew Ostor Speakers bureau: AÖ has received speaker or consulting fees and/or research grants from AbbVie, Bristol-Myers Squibb, Celgene, Janssen, Lilly, Merck, Novartis, Pfizer, Roche, Sanofi, and UCB., Consultant of: AÖ has received speaker or consulting fees and/or research grants from AbbVie, Bristol-Myers Squibb, Celgene, Janssen, Lilly, Merck, Novartis, Pfizer, Roche Sanofi, and UCB., Grant/research support from: AÖ has received speaker or consulting fees and/or research grants from AbbVie, Bristol-Myers Squibb, Celgene Janssen, Lilly, Merck, Novartis, Pfizer, Roche, Sanofi, and UCB., Filip van den Bosch Speakers bureau: FVdB has received speaker and/or consulting fees from Abbvie Celgene, Galapagos, Gilead, Janssen, Lilly, Novartis, Pfizer, and UCB., Consultant of: FVdB has received speaker and/or consulting fees from Abbvie, Celgene Galapagos, Gilead, Janssen, Lilly, Novartis, Pfizer, and UCB., Kim Papp Speakers bureau: KP has received honoraria or fees for serving on advisory boards, as a speaker, and as a consultant, as well as grants as principal investigator from AbbVie, Amgen, Astellas, Bausch Health (Valeant), Baxalta, Baxter, Boehringer Ingelheim, Bristol-Myers Squibb, Celgene, Coherus, Dermira, EMD Serono, Forward Pharma Galderma, Genentech, GlaxoSmithKline, Janssen, Kyowa Kirin, LEO Pharma, Lilly, Medlmmune, Merck, Novartis, Pfizer, Regeneron, Roche, Sanofi Genzyme Stiefel, Sun Pharma, Takeda, and UCB., Consultant of: KP has received honoraria or fees for serving on advisory boards, as a speaker, and as a consultant, as well as grants as principal investigator from AbbVie, Amgen, Astellas, Bausch Health (Valeant), Baxalta, Baxter, Boehringer Ingelheim, Bristol-Myers Squibb, Celgene Coherus, Dermira, EMD Serono, Forward Pharma, Galderma, Genentech, GlaxoSmithKline, Janssen, Kyowa Kirin, LEO Pharma, Lilly, Medlmmune, Merck, Novartis, Pfizer, Regeneron, Roche, Sanofi Genzyme, Stiefel, Sun Pharma, Takeda, and UCB., Grant/research support from: KP has received honoraria or fees for serving on advisory boards, as a speaker, and as a consultant, as well as grants as principal investigator from AbbVie, Amgen, Astellas, Bausch Health (Valeant), Baxalta, Baxter, Boehringer Ingelheim, Bristol-Myers Squibb, Celgene, Coherus, Dermira EMD Serono, Forward Pharma, Galderma, Genentech, GlaxoSmithKline, Janssen, Kyowa Kirin, LEO Pharma, Lilly, Medlmmune, Merck, Novartis, Pfizer, Regeneron, Roche, Sanofi Genzyme, Stiefel, Sun Pharma, Takeda, and UCB., CECILIA ASNAL Speakers bureau: CA has received honoraria or fees for serving on advisory boards or as a speaker, as well as research support from AbbVie, Genentech, Janssen, Lilly, Pfizer, and Roche., Grant/research support from: CA has received honoraria or fees for serving on advisory boards or as a speaker, as well as research support from AbbVie, Genentech, Janssen, Lilly, Pfizer, and Roche., Ricardo Blanco Speakers bureau: RB has received grants or research support from AbbVie, Merck, and Roche; and has received consultation fees or honoraria for serving as a speaker for AbbVie, Bristol-Myers Squibb, Janssen, Lilly, Merck, Pfizer, and Roche., Consultant of: RB has received grants or research support from AbbVie, Merck, and Roche; and has received consultation fees or honoraria for serving as a speake for AbbVie, Bristol-Myers Squibb, Janssen, Lilly, Merck, Pfizer, and Roche., Grant/ 
research support from: RB has received grants or research support from AbbVie, Merck, and Roche; and has received consultation fees or honoraria for serving as a speaker for AbbVie, Bristol-Myers Squibb, Janssen, Lilly, Merck, Pfizer, and Roche. Jacob Aelion Grant/research support from: JA has received grants or research support from AbbVie, Amgen, AstraZeneca, Bristol-Myers Squibb, Galapagos/Gilead Genentech, GlaxoSmithKline, Lilly, Mallinckrodt, Nektar Therapeutics, Nichi-lko, Novartis, Pfizer, Regeneron, Roche, Sanofi, Selecta Biosciences, and UCB., Gabriela Alperovich Shareholder of: GA is a full-time employee of AbbVie, and may hold AbbVie stock or stock options., Employee of: GA is a full-time employee of AbbVie, and may hold AbbVie stock or stock options., Ying Zhang Shareholder of: $Y Z$ is a former AbbVie employee, and may hold AbbVie stock or stock options., Employee of: $Y Z$ is a former AbbVie employee, and may hold AbbVie stock or stock options., Zailong Wang Shareholder of: ZW is a full-time employee of AbbVie, and may hold AbbVie stock or stock options., Employee of: ZW is a full-time employee of AbbVie, and may hold AbbVie stock or stock options., Ahmed M. Soliman Shareholder of: AS is a full-time employee of AbbVie, and may hold AbbVie stock or stock options., Employee of: AS is a full-time employee of AbbVie, and may hold AbbVie stock or stock options., Ann Eldred Shareholder of: AE is a full-time employee of AbbVie, and may hold AbbVie stock or stock options., Employee of: AE is a full-time employee of AbbVie, and may hold AbbVie stock or stock options., Alan Kivitz Shareholder of: AK is a shareholder of or has received honoraria or fees as a consultant, speaker, or expert witness for AbbVie, Boehringer Ingelheim, Celgene, Flexion, Gilead, GlaxoSmithKline, Janssen, Lilly, Merck, Novartis, Pfizer, Regeneron, Sanofi Genzyme, Sun Pharma, and UCB., Speakers bureau: AK is a shareholder of or has received honoraria or fees as a consultant, speaker, or expert witness for AbbVie, Boehringer Ingelheim, Celgene, Flexion, Gilead, GlaxoSmithKline, Janssen, Lilly, Merck, Novartis, Pfizer, Regeneron, Sanofi Genzyme, Sun Pharma, and UCB., Consultant of: AK is a shareholder of or has received honoraria or fees as a consultant, speaker, or expert witness for AbbVie, Boehringer Ingelheim, Celgene, Flexion, Gilead, GlaxoSmithKline, Janssen, Lilly, Merck, Novartis, Pfizer, Regeneron, Sanofi Genzyme, Sun Pharma, and UCB.

DOI: 10.1136/annrheumdis-2021-eular.2695

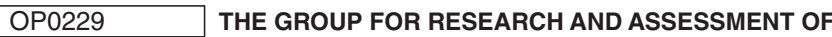 PSORIASIS AND PSORIATIC ARTHRITIS (GRAPPA) TREATMENT RECOMMENDATIONS 2021}

L. C. Coates ${ }^{1}$, E. Soriano ${ }^{2}$, N. Corp ${ }^{3}$, H. Bertheussen ${ }^{4}$, K. Callis-Duffin ${ }^{5}$ C. Barbosa Campanholo ${ }^{6}$, J. Chau $^{7}$, L. Eder ${ }^{8}$, D. Fernandez ${ }^{9}$, O. Fitzgerald ${ }^{10}$, A. Garg ${ }^{11}$, D. D. Gladman ${ }^{12}$, N. Goel ${ }^{13,14}$, S. Grieb ${ }^{15}$, P. Helliwell ${ }^{16}$, M. E. Husni ${ }^{17}$ D. Jadon ${ }^{18}$, A. Katz ${ }^{19}$, D. Laheru ${ }^{20}$, J. Latella ${ }^{21}$, Y. Y. Leung ${ }^{22}$, C. Lindsay ${ }^{23}$, E. Lubrano ${ }^{24}$, L. Mazzuoccolo ${ }^{25}$, R. Mcdonald ${ }^{26}$, P. J. Mease ${ }^{27,28}$, D. O'sullivan ${ }^{29}$ A. Ogdie ${ }^{30}$, W. Olsder ${ }^{31}$, L. Schick ${ }^{15}$, I. Steinkoenig ${ }^{32}$, M. De Wit ${ }^{33}$, D. Van der Windt ${ }^{34}$, A. Kavanaugh ${ }^{35} .{ }^{1}$ University of Oxford, NDORMS, Oxford, United Kingdom; ${ }^{2}$ Hospital Italiano de Buenos Aires, Rheumatology, Buenos Aires, Argentina; ${ }^{3}$ Keele University, School of Primary, Community and Social Care, Keele, United Kingdom; ${ }^{4}$ GRAPPA, Patient Research Partner, Oslo, Norway; ${ }^{5}$ University of Utah, Dermatology, Salt Lake City, United States of America; ${ }^{6}$ Santa Casa de Sao Paulo, Rheumatology, Sao Paulo, Brazil; ${ }^{7}$ GRAPPA, Patient Research Partner, Hong Kong, Hong Kong (SAR); ${ }^{8}$ University of Toronto, Rheumatology, Toronto, Canada; ${ }^{9}$ Hospital Universitario San Ignacio, Rheumatology, Bogota, Colombia; ${ }^{10}$ University College Dublin, Rheumatology, Dublin, Ireland; ${ }^{11}$ Donald and Barbara Zucker School of Medicine at Hofstral Northwell, Dermatology, Hempstead, United States of America; ${ }^{12}$ Toronto Western Hospital, Schroeder Arthritis Institute, Toronto, Canada; ${ }^{13} \mathrm{GRAPPA}$, Patient Research Partner, Durham, United States of America; ${ }^{14}$ Duke University, Rheumatology, Durham, United States of America; ${ }^{15}$ GRAPPA, Patient Research Partner, Seattle, United States of America; ${ }^{16}$ University of Leeds, Leeds Institute of Rheumatology and Musculoskeletal Medicine, Leeds, United Kingdom; ${ }^{17}$ Cleveland Clinic Main Campus, Rheumatology, Cleveland, United States of America; ${ }^{18}$ University of Cambridge, Rheumatology, Cambridge, United Kingdom; ${ }^{19} \mathrm{GRAPPA}$, Patient Research Partner, Haifa, Israel; ${ }^{20} \mathrm{Ch}$ urchill Hospital, Dermatology, Oxford, United Kingdom; ${ }^{21}$ GRAPPA, Patient Research Partner, Connecticut, United States of America; ${ }^{22}$ Singapore General Hospital, Rheumatology, Singapore, Singapore; ${ }^{23}$ GRAPPA, Patient Research Partner, Propser, United States of America; ${ }^{24}$ University of Molise, Rheumatology, Campobasso, Italy; ${ }^{25}$ Hospital Italiano de Buenos Aires, Dermatology, Buenos Aires, Argentina; ${ }^{26}$ GRAPPA, Patient Research Partner, Toronto, Canada; ${ }^{27}$ Swedish Medical Center, Rheumatology, Seattle, United States of America; ${ }^{28}$ University of Washington, Rheumatology, Seattle, United States of America; ${ }^{29}$ GRAPPA, Patient Research Partner, Dublin, Ireland; ${ }^{30}$ University of Pennsylvania, Rheumatology, Philadelphia, United States of America; ${ }^{31}$ GRAPPA, Patient Research Partner, Eindhoven, Netherlands; ${ }^{32}$ GRAPPA, Patient Research Partner, Cleveland, United States of America; ${ }^{33}$ GRAPPA, Patient Research Partner, Amsterdam, Netherlands; ${ }^{34}$ Keele University, School of Primary Community and Social Care, Keele, United Kingdom; ${ }^{35}$ University of California San Diego, Rheumatology, La Jolla, United States of America
Background: Since the 2015 GRAPPA treatment recommendations were published, therapeutic options and management strategies for psoriatic arthritis (PsA) have advanced considerably.

Objectives: The goal of the GRAPPA recommendations update is to develop high quality, evidence-based recommendations for the treatment of PsA, including related conditions and comorbidities.

Methods: GRAPPA rheumatologists, dermatologists and patient research partners (PRPs) updated overarching principles for the management of adults with PsA by consensus. Principles considering use of biosimilars and tapering/discontinuing of therapy were added to this update. Systematic literature searches based on data publicly available from three databases (MEDLINE, EMBASE, and Cochrane CENTRAL) were conducted from the end of the previous recom mendations' searches through August 2020. Additional abstract searches were performed for conference presentations in 2017-2020. Searches covered PsA treatments (peripheral arthritis, axial arthritis, enthesitis, dactylitis, skin, and nail disease). Additional searches were performed for related conditions (uveitis and IBD) and comorbidities evaluating their impact on safety and treatment outcomes. Individual groups assessed the risk of bias and applied the GRADE system to generate strong or conditional recommendations for therapies within the domain groups and for the management of comorbidities and related conditions. These recommendations were then incorporated into an overall treatment schema.

Results: Updated, evidence-based treatment recommendations are shown (Table 1). Since 2015, many new medications have been incorporated. Additiona results for older medications, such as methotrexate, have been published across PsA domains. Based on the evidence, the treatment recommendations developed by individual groups were incorporated into the overall schema including principles for management of arthritis, spondylitis, enthesitis, dactylitis, skin, and nail disease in PsA, and associated conditions (Figure 1). Choice of therapy for an individual should ideally address all of the domains that impact on that patient, supporting shared decision making with the patient involved. Additional consideration in the recommendations was given to key associated conditions and comorbidities as these often impact on therapy choice.

Conclusion: These GRAPPA treatment recommendations provide up to date, evidence-based guidance to providers who manage and treat adult patients with PSA These recommendations are based on domain-based strategy for PsA and supplemented by overarching principles developed by consensus of GRAPPA members.

\begin{tabular}{|c|c|c|c|c|c|}
\hline Indication & $\begin{array}{l}\text { Strong } \\
\text { For }\end{array}$ & $\begin{array}{l}\text { Conditional } \\
\text { For }\end{array}$ & $\begin{array}{l}\text { Conditional } \\
\text { Against }\end{array}$ & $\begin{array}{c}\text { Strong } \\
\text { Against }\end{array}$ & $\begin{array}{l}\text { Insufficient } \\
\text { evidence }\end{array}$ \\
\hline $\begin{array}{l}\text { Peripheral } \\
\text { Arthritis } \\
\text { DMARD } \\
\text { Naïve }\end{array}$ & $\begin{array}{l}\text { csDMARDs, } \\
\text { TNFi, PDE4i, } \\
\text { IL-12/23i, IL-17i, } \\
\text { IL-23i, JAKi }\end{array}$ & $\begin{array}{l}\text { NSAIDs, } \\
\text { oral CS, IA CS, }\end{array}$ & IL-6i, & & \\
\hline $\begin{array}{l}\text { Peripheral } \\
\text { Arthritis } \\
\text { DMARD } \\
\text { IR }\end{array}$ & $\begin{array}{l}\text { TNFi, IL-12/23i, } \\
\text { IL-17i, IL-23i, } \\
\text { JAKi }\end{array}$ & $\begin{array}{l}\text { PDE4i, other } \\
\text { csDMARD, } \\
\text { NSAIDs, oral } \\
\text { CS, IA CS, }\end{array}$ & IL-6i, & & \\
\hline $\begin{array}{l}\text { Peripheral } \\
\text { Arthritis }\end{array}$ & $\begin{array}{l}\text { TNFi, IL-17i, } \\
\text { IL-23i, JAKi, }\end{array}$ & $\begin{array}{l}\text { NSAIDs, oral } \\
\text { CS, IA CS, } \\
\text { IL-12/23i, PDE4i, }\end{array}$ & IL-6i, & & \\
\hline bDMARD IR & & CTLA-4-Ig & & & \\
\hline $\begin{array}{l}\text { Axial } \\
\text { arthritis, } \\
\text { Biologic } \\
\text { Naïve }\end{array}$ & $\begin{array}{l}\text { NSAIDs, } \\
\text { Physiotherapy, } \\
\text { simple analgesia, } \\
\text { TNFi, IL-17i, JAKi }\end{array}$ & $\begin{array}{l}\text { CS SIJ injections, } \\
\text { bisphosphonates }\end{array}$ & & $\begin{array}{l}\text { Cs } \\
\text { DMARDs, } \\
\text { IL-6i, }\end{array}$ & IL-12/23i, IL-23i \\
\hline $\begin{array}{l}\text { Axial PsA, } \\
\text { Biologic } \\
\text { IR }\end{array}$ & $\begin{array}{l}\text { NSAIDs, } \\
\text { Physiotherapy, sim- } \\
\text { ple analgesia, TNFi, } \\
\text { IL-17i, JAKi }\end{array}$ & & & $\begin{array}{l}\text { csD- } \\
\text { MARDs, } \\
\text { IL-6i, }\end{array}$ & IL-12/23i, IL-23i \\
\hline Enthesitis & $\begin{array}{l}\text { TNFi, IL-12/23i, } \\
\text { IL-17i, PDE4i, } \\
\text { IL-23i, JAKi }\end{array}$ & $\begin{array}{l}\text { NSAIDs, phys- } \\
\text { iotherapy, CS } \\
\text { injections, MTX }\end{array}$ & & IL-6i, & $\begin{array}{l}\text { Other cs } \\
\text { DMARDs }\end{array}$ \\
\hline Dactylitis & $\begin{array}{l}\text { TNFi IL-12/23i, } \\
\text { IL-17i, IL-23i, } \\
\text { JAKi, PDE4i }\end{array}$ & $\begin{array}{l}\text { NSAIDs, CS } \\
\text { injections, MTX }\end{array}$ & $\begin{array}{l}\text { Other } \\
\text { csDMARDs }\end{array}$ & & \\
\hline Psoriasis & $\begin{array}{l}\text { Topicals, photother- } \\
\text { apy, csDMARDs, } \\
\text { TNFi, IL-12/23i, } \\
\text { IL-17i, IL-23i, } \\
\text { PDE4i, JAKi }\end{array}$ & Acitretin & & & \\
\hline $\begin{array}{l}\text { Nail } \\
\quad \text { psoriasis }\end{array}$ & $\begin{array}{l}\text { TNFi, IL12/23i, } \\
\text { IL17i, IL23i, } \\
\text { PDE4i }\end{array}$ & $\begin{array}{l}\text { Topical CS, } \\
\text { tacrolimus and } \\
\text { calcipotriol } \\
\text { combination or } \\
\text { individual ther- } \\
\text { apies, Pulsed } \\
\text { dye laser, } \\
\text { csDMARDs, } \\
\text { acitretin, JAKi }\end{array}$ & & & $\begin{array}{l}\text { Topical } \\
\text { Cyclosporine } \\
\text { /Tazarotene, } \\
\text { Fumarate, } \\
\text { Fumaric Acid } \\
\text { Esters, UVA } \\
\text { and UVB } \\
\text { Phototherapy, } \\
\text { Alitretinoin }\end{array}$ \\
\hline IBD & $\begin{array}{l}\text { TNFi (not ETN), } \\
\text { IL-12/23i, JAKi }\end{array}$ & & & IL-17i & \\
\hline Uveitis & TNFi (not ETN) & & & & \\
\hline
\end{tabular}

